\title{
Impact of HIV on mortality from acute lower respiratory tract infection in rural Zambia
}

\author{
A Smyth, C Y W Tong, H Carty, C A Hart
}

\begin{abstract}
Aims-To establish the prevalence and clinical correlates of HIV among children with acute lower respiratory tract infection.

Methods-Children admitted to a rural Zambian hospital were studied over an eight month period. The diagnosis of acute lower respiratory tract infection was made clinically, according to World Health Organisation (WHO) criteria. Clinicians, who were unaware of the children's HIV status, prescribed antibiotic and supportive treatment according to WHO guidelines. HIV status was established using the polymerase chain reaction (Amplicor HIV1, Roche) applied to dried blood spots.
\end{abstract}

Results-Acute lower respiratory tract infection was diagnosed in 132 children (median age 8 months, range 1 month to 4 years). The WHO criteria for severe or very severe pneumonia were met by $96 / 132$ patients $(73 \%)$ and 21 patients $(16 \%)$ died. HIV dried blood spot PCR was positive in 14 cases $(11 \%)$, of whom four fulfilled the WHO clinical case definition for paediatric AIDS and five died. The group as a whole were malnourished, but the HIV positive children were more severely malnourished (mean $\mathrm{z}$ score for weight = -3.01) than the HIV negative children (mean $\mathrm{z}$ score $=-1.73, \mathrm{p}<0.001)$. The relative risk of death was 2.6 in the HIV positive group but this was not significant $(\mathrm{p}=0.079)$.

St Francis Hospital, Katete, Zambia A Smyth

Department of Medical Microbiology, Royal Liverpool

University Hospital, Liverpool

C Y W Tong

C A Hart

Royal Liverpool Children's Hospital Alder Hey, Liverpool H Carty

Correspondence to: Dr Alan Smyth, Department of Paediatrics, Nottingham City Hospital, Hucknall Road, Nottingham NG5 1PB.

Accepted 11 June 1997
Conclusions-An important minority of children with acute lower respiratory tract infection in rural Zambia will be infected with HIV. However, most HIV positive children presenting with respiratory infection will survive given simple antibiotic and supportive treatment. (Arch Dis Child 1997;77:227-230)

Keywords: HIV; acute lower respiratory tract infection; polymerase chain reaction; malnutrition

Acute lower respiratory tract infection is responsible for the deaths of four million children under 5 each year. Most of these deaths occur in developing countries and most are in children under 12 months old. ${ }^{1}$ It has been suggested that a high proportion of children with acute lower respiratory tract infection may have be infected with the HIV virus, acquired by vertical transmission. ${ }^{23}$
The diagnosis of HIV infection is difficult in children under 12 months of age. The World Health Organisation (WHO) has proposed a clinical case definition for paediatric AIDS $^{4}$ but this has been shown to have a low sensitivity. ${ }^{5}$ Serological methods of testing for HIV are unreliable due to the possibility of transplacental transmission of antibodies from the seropositive mother. ${ }^{6}$ The use of the polymerase chain reaction (PCR), which detects HIV proviral DNA, has been established as a reliable method of identifying true HIV infection in young infants. ${ }^{7}$ This may be performed on whole blood or dried blood spots on filter paper. Dried blood spots are easy to collect, require only a small volume of blood, and store well at ambient temperatures. ${ }^{8}$

The purpose of this study was to establish the prevalence and clinical correlates of HIV infection in a group of children with acute lower respiratory tract infection. We have employed the polymerase chain reaction for HIV proviral DNA on dried blood spots, collected on filter paper.

\section{Methods}

Patients with acute lower respiratory tract infection, admitted to the paediatric ward at St Francis Hospital over an eight month period from November 1994 to June 1995, were studied. The admission criteria for children with respiratory infections were severe or very severe pneumonia according to WHO guidelines ${ }^{9}$ (table 1), or pneumonia with another indication for hospital admission, for example severe malnutrition. Less severely ill children were treated as outpatients and were not eligible for entry into the study. Only children under 5 years were admitted to the children's ward. Children under 4 weeks of age were not studied because of the difficulty of making a clinical diagnosis in this age group and to reduce the possibility of false negative results on PCR. Children were excluded if widespread wheeze was audible on auscultation of the chest or if their parents took them away from the hospital before treatment was completed.

Patients were examined clinically within 24 hours of admission by one of us (AS). Clinical features described in the WHO definition of severe and very severe pneumonia were noted ${ }^{9}$ (table 1), as were findings required for the WHO clinical case definition of paediatric AIDS $^{4}$ (table 2). Each child was weighed and the oxygen saturation recorded using an Ohmeda Biox 3740 pulse oximeter (Ohmeda, Louisville, USA). The normal range for oxygen saturation at this altitude $(1150 \mathrm{~m})$ had previously 
Table 1 WHO clinical criteria for very severe and severe pneumonia

For the child age 2 months up to 5 years with cough or difficult breathing (who does not have stridor, severe undernutrition, or signs suggesting meningitis)

^Very severe pneumonia $\quad$ Central cyanosis

$\begin{array}{ll}\star \text { Severe pneumonia } & \text { Not able to drink } \\ & \text { No central cyanosis and able to drink but chest indrawing }\end{array}$

^Admission advised for both these categories

All patients with very severe pneumonia should receive oxygen. Where supply of oxygen is ample, those with severe pneumonia should receive oxygen if any of the following clinical signs are present: restlessness (if oxygen improves the condition); severe chest indrawing; breathing rate of 70 breaths/min or more.

Table 2 WHO clinical case definition for paediatric AIDS

\begin{tabular}{ll}
\hline Major signs & Weight loss or failure to thrive \\
& Chronic diarrhoea $(>1$ month) \\
& Prolonged fever $(>1$ month) \\
Minor signs & Generalised lymphadenopathy \\
& Oropharyngeal candidiasis \\
& Repeated common infections \\
& (otitis, pharyngitis, etc) \\
& Persistent cough $(>1$ month) \\
& Generalised dermatitis \\
& Confirmed maternal HIV infection
\end{tabular}

Paediatric AIDS is suspected in an infant or child presenting with at least two major signs associated with at least two minor signs in the absence of known causes of immunosuppression. ${ }^{\star}$ Generalised lymphadenopathy $=1 \mathrm{ymph}$ nodes measuring at least $0.5 \mathrm{~cm}$ and present in two or more sites, with bilateral lymph nodes counting as one site.

been established by studying 85 asymptomatic children; an oxygen saturation below the 2.5 centile $(92 \%)$ was taken to indicate hypoxaemia. Oxygen was given to those children who had an oxygen saturation less than $92 \%$.

Antibiotics were given according to WHO guidelines. ${ }^{9}$ Laboratory facilities for microbiology were very limited, and blood and sputum culture were not performed. Chest radiographs were taken when the clinical diagnosis was uncertain or when complications, such as empyema, were thought to have occurred. Where clinical response to antibiotics was poor after seven days and the chest radiograph was suggestive of primary tuberculosis, then an empirical trial of antituberculous chemotherapy was begun. All chest radiographs were later examined by a radiologist (HC) who was not aware of the clinical features or the HIV status of the child.

Blood is routinely collected from all children admitted to the paediatric ward at St Francis Hospital for haemoglobin estimation and thick blood film for malaria parasites. A $50 \mu \mathrm{l}$ aliquot of blood from this routine specimen was used to prepare a dried blood spot, using standard newborn screening papers (Schleicher and Schuell 903). These were allocated a code number and stored in $1.5 \mathrm{ml}$ sealed plastic tubes (Sarstedt) at room temperature. Samples were returned to the United Kingdom for analysis. All records relating to children in this study were identified by a code number only.

The method used for the HIV PCR was as described by Cassol et $a l^{7}$ and makes use of a commercial PCR kit - the Amplicor HIV-1 kit (Roche Diagnostic Systems, New Jersey, USA) to amplify a sequence on a highly conserved region of the gag gene. Briefly, this uses a chelex extraction, after removal of haemoglobin from the sample. The amplification step uses biotinylated primers (Bio-SK431 and BioSK462) and 35 amplification cycles. The PCR product is detected by hybridisation to a DNA probe (SK102) bound to a microwell plate and uses an avidin-horseradish peroxidase conjugate to produce a colour change. This is quantified using a microwell plate reader, with a cut off value for optical density of 0.35 or greater $A_{450}$ units. A serial dilution was performed with HIV seronegative blood 'spiked' with 8E5 cells - a cell line containing one copy of HIV proviral DNA per cell. This revealed that the assay could detect one copy of HIV proviral DNA per $6 \mathrm{~mm}$ filter paper disc. One $6 \mathrm{~mm}$ filter paper disc was analysed per patient and any equivocal results were repeated.

Statistical analysis was by means of the $\chi^{2}$ test and $t$ test for unpaired data, using the Arcus Pro-stat statistical package; z scores for weight were calculated using the 'Epi Nut' program on the 'Epi Info' statistical package.

Ethical permission for the study was given by the ethics committee of St Francis Hospital and by the research ethics committee of the Royal Liverpool Children's Hospital NHS Trust.

\section{Results}

Over the eight month study period, 139 patients had a clinical diagnosis of acute lower respiratory infection. Wheeze was present in three patients, and four were taken away by their parents before treatment was complete. These patients were excluded from the study, leaving 132 patients. There were 64 boys $(48 \%)$ and the median age was 8 months (range 1 month to 4 years). The WHO criteria for severe or very severe pneumonia ${ }^{9}$ were fulfilled by 96 patients (73\%). There were 21 deaths (overall mortality rate $16 \%$ ). A chest radiograph was taken in 84 patients, and unilateral or bilateral lobar or segmental consolidation was present in $68(81 \%)$.

HIV dried blood spot PCR was positive in 14 cases $(11 \%)$, of which only four fulfilled the WHO clinical case definition for paediatric AIDS and five died. The WHO clinical case definition was also fulfilled by three children who were HIV PCR negative. Table 3 shows the frequency with which each of the clinical features in the WHO definition was found both in the HIV PCR positive and HIV PCR negative groups. Three mothers had previously been identified as HIV positive when serological testing was performed for clinical indica-

Table 3 Frequency of occurrence of the clinical features of HIV infection (WHO clinical case definition) in HIV PCR positive and HIV PCR negative groups

\begin{tabular}{|c|c|c|c|c|c|c|c|c|c|}
\hline & $\begin{array}{l}\text { Failure to } \\
\text { thrive }\end{array}$ & $\begin{array}{l}\text { Diarrhoea } \\
\text { (>4 weeks) }\end{array}$ & $\begin{array}{l}\text { Fever } \\
(>4 \text { weeks) }\end{array}$ & Lymphadenopathy & $\begin{array}{l}\text { Oral } \\
\text { candida }\end{array}$ & $\begin{array}{l}\text { Repeated } \\
\text { common } \\
\text { infections }\end{array}$ & $\begin{array}{l}\text { Cough } \\
\text { (>4 weeks) }\end{array}$ & $\begin{array}{l}\text { Generalised } \\
\text { dermatitis }\end{array}$ & $\begin{array}{l}\text { Maternal } \\
\text { HIV }\end{array}$ \\
\hline HIV PCR positive $(\mathrm{n}=14)$ & 11 & 3 & 6 & 1 & 3 & 6 & 6 & 0 & 3 \\
\hline HIV PCR negative $(n=118)$ & 67 & 12 & 17 & 0 & 12 & 7 & 25 & 0 & 0 \\
\hline
\end{tabular}


tions. Their children were HIV PCR positive. The clinical case definition had a low sensitivity when compared with HIV PCR (sensitivity $31 \%$, specificity $98 \%$, positive predictive value $57 \%)$.

The HIV PCR positive and HIV PCR negative groups did not show significant differences in either age or gender. The two groups were similar when comparing the proportion from each group who fell into the categories of severe or very severe pneumonia, who had chest radiograph abnormalities consistent with pneumonia, and who were hypoxaemic. The chest radiographs of two children showed reticulonodular infiltrates consistent with lymphoid interstitial pneumonitis. Both were HIV PCR positive.

The group as a whole were malnourished (mean $\mathrm{z}$ score for weight $=-1.86$ ). The HIV positive children were considerably more malnourished (mean $\mathrm{z}$ score $=-3.01$ ) than the HIV negative group (mean $\mathrm{z}$ score $=-1.73$ ). The difference between the means was 1.28 (95\% confidence interval -1.98 to $-0.58, \mathrm{p}<$ 0.001 when the unpaired two sample $t$ test was applied).

Of those patients who were HIV PCR positive, five of $14(36 \%)$ died compared with 16 of 118 (14\%) who were HIV PCR negative. The odds ratio for death in the HIV PCR positive patients was 2.6 but this was not significant $(\mathrm{p}=0.079)$.

Among the HIV PCR positive children, three of $14(21 \%)$ were started empirically on a trial of antituberculous chemotherapy, compared with 11 of 118 (9\%) of the HIV PCR negative group. However, the difference was not statistically significant $\left(\chi^{2}=0.35, \mathrm{p}=0.351\right)$. Other complications observed were pleural effusion, empyema, pneumothorax, pneumomediastinum, and pyogenic pericarditis. The numbers with each complication were small and in none of these was there a significant difference between the HIV positive and HIV negative groups.

\section{Discussion}

This study has shown that, in a rural hospital in Africa, the prevalence of HIV in children with acute lower respiratory tract infection is approximately $11 \%$. This is, to our knowledge, the first time the PCR has been used to establish the prevalence of HIV among a group of African children with acute lower respiratory tract infection. The majority of children enrolled in this study were malnourished, but the HIV positive children were more malnourished than their HIV negative peers. Although the risk of death from pneumonia was increased by more than twofold in the HIV positive children, the majority survived when given simple antibiotic and supportive treatment.

The prevalence of antibodies to HIV among women living in rural areas in Zambia is known to be around $13 \% .{ }^{10}$ The rate of vertical transmission of HIV from mother to infant in Zambia is approximately $39 \% .{ }^{11}$ Therefore the prevalence of vertically acquired HIV infection among young children in rural Zambia would be expected to be around $5 \%$. The relative risk of HIV among children admitted to hospital with acute respiratory infection in rural Zambia is therefore approximately twice what would be expected in the background population.

Lepage et al studied Rwandan children and found the sensitivity of the WHO clinical case definition for paediatric AIDS ${ }^{4}$ was low. ${ }^{5}$ They proposed a simplified clinical case definition in which respiratory distress secondary to lower respiratory infection was an AIDS defining feature. All the children described in the present study had some degree of respiratory distress secondary to lower respiratory tract infection and $73 \%$ had severe or very severe pneumonia by WHO criteria. ${ }^{9}$ However, only $11 \%$ had evidence of HIV infection as determined by PCR, and so the simplified clinical case definition proposed by Lepage et al performs poorly in our study population (specificity $=0 \%$ ).

Chintu et al studied the seroprevalence of HIV in 1266 children admitted to hospital in Lusaka, the Zambian capital. ${ }^{2}$ They found that the overall prevalence of HIV in paediatric admissions was $28 \%$ and that the rate was the same among the subgroup of children with pneumonia. This prevalence is much higher than the rates of $11 \%$ found, using PCR, in our study. However, the seroprevalence of HIV among women of childbearing age in Zambian cities is approximately $30 \%,{ }^{12}$ whereas in rural Zambia the rate is around $13 \% .^{10}$ The lower prevalence of HIV in children found in our study is therefore in proportion to the lower seroprevalence seen in the adult population in rural areas. Chintu et al also found a high seroprevalence of HIV among children with malnutrition (41\%). Mortality from acute lower respiratory infection was similar in children who were HIV positive $(11 \%)$ and HIV negative $(8.4 \%)$ in their study.

Nathoo $e t a l,{ }^{3}$ in Zimbabwe, found clinical or serological evidence of HIV in $31 \%$ of urban children with acute lower respiratory tract infection, and moderate to severe malnutrition (weight for age $<80 \%$ ) in $30 \%$. The case fatality rate was $16 \%$ and the risk of death was increased over threefold in children who had evidence of HIV infection. In our study $71 \%$ of the children were less than $80 \%$ of weight for age. The reason for such poor nutrition is obscure. Chronic diarrhoea occurred in only $15 / 131$ patients $(11 \%)$ (table 3 ) and was no more common in the HIV positive group. However, in Katete district alone over 2400 children $(3.4 \%)$ have lost both parents to HIV related illness (JM Cairns, personal communication). Although these children are cared for by the extended family, the financial strain is considerable and they may be at greater risk of malnutrition as a result. We observed similar mortality rates to the children studied in Zimbabwe, and a similar increase in mortality in the HIV positive group.

The present study employed the polymerase chain reaction, using dried blood spots, to detect infection with HIV. This method has been shown to be highly sensitive (detecting $99.4 \%$ of infants vertically infected with HIV 
when the sample is taken after 15 days of age) and specific (specificity $=100 \%) .{ }^{7}$ An assay of dried blood spots 'spiked' with $8 \mathrm{E} 5$ cells, performed as part of the present study, showed that one copy of HIV proviral DNA could be detected in a $6 \mathrm{~mm}$ filter paper punch. As the target sequence is a highly conserved region, the probability of false negative results due to strain variation is low. Dried blood spot samples are robust. Storage at $22^{\circ} \mathrm{C}$ for three months and freeze-thawing twice have been shown to have no effect on PCR reactivity. ${ }^{8}$ In the present study samples were stored for up to eight months at temperatures of around $22^{\circ} \mathrm{C}$ and it is possible that some loss of PCR reactivity and reduction in the sensitivity of the assay may have occurred. However, there are no published data on storage for longer periods.

In the first year of life, the possibility of transplacental transmission of antibodies to HIV makes the use of HIV serology unreliable. Although $75 \%$ of infants will lose maternal antibody to HIV by 1 year of age, a significant proportion of children who become antibody negative will be infected with HIV. ${ }^{6}$ HIV PCR represents a more reliable way of making the diagnosis of HIV infection in young children.

This study used clinical criteria to diagnose acute lower respiratory tract infection ${ }^{9}$ and this may have lead to diagnostic imprecision. However, chest radiographs are expensive to perform in the developing world. Most health workers will have to make this diagnosis on clinical grounds alone and so it seemed appropriate to use clinical criteria in this study. The observation that consolidation was present in $81 \%$ cases where a chest radiograph was taken suggests that the clinical diagnosis was correct in most cases.

We did not study children over the age of 5 years. The oldest child found to be HIV PCR positive was aged 3 years, and the median age of the HIV PCR positive children was 11 months. Of the children over 1 year, three of six died while still hospital inpatients. In a serosurvey of children in hospital in Kampala, Uganda, ${ }^{13} 80 \%$ of HIV positive children were under 2 years and $23 \%$ died within three months. Although we found that HIV infected children had a good response to conventional treatment for pneumonia, it seems unlikely that many children will survive to reach their fifth birthday. Long term follow up data are sorely needed on children vertically infected with HIV in the developing world.

In conclusion, an important minority of children with acute lower respiratory tract infection in rural Zambia will be infected with HIV. However, most HIV positive children presenting with respiratory infection will survive given simple antibiotic and supportive treatment. Malnutrition was a more severe problem in the HIV infected group and more research is needed to establish whether intensive nutritional rehabilitation can reduce mortality in these children. Further work now needs to be done to establish if the pathogens associated with acute lower respiratory tract infection in the developing world have changed in association with the HIV epidemic.

This work was funded by grants from the Paediatric Research Society, British Paediatric Association, the Royal Liverpool Childrens' Hospital Research and Development Fund, and the Royal Liverpool Childrens' Hospital Respiratory Unit.

1 World Health Organisation. World health report. Geneva: WHO, 1995.

2 Chintu C, Luo C, Bhat G, et al. Impact of human immunodeficiency virus type-1 on common pediatric illnesses in Zambia. F Trop Pediatr 1995;41:348-53.

3 Nathoo KJ, Nkrumah FK, Ndlovu D, Nhembe M, Pirie DJ, Kowo $\mathrm{H}$. Acute lower respiratory tract infection in hospitalised children in Zimbabwe. Ann Trop Paediatr 1993;13:253-61.

4 World Health Organisation. Acquired immunodeficiency syndrome (AIDS): WHO/CDC case definition for AIDS. Wkly Epidemiol Rec 1986;61:69-73.

5 Lepage P, van de Perre P, Dabis F, et al. Evaluation and simplification of the World Health Organisation clinical case plification of the World Health Organisation clinical
definition for paediatric AIDS. AIDS 1989;3:221-5.

6 Mok JQ, Giaquinto C, De Rossi A, Grosch-Wörner I, Ades $\mathrm{AE}$, Peckham CS. Infants born to mothers seropositive for human immunodeficiency virus. Preliminary findings from a multicentre European study. Lancet 1987;i:1164-8.

7 Cassol S, Butcher A, Kinard S, et al. Rapid screening for early detection of mother-to-child transmission of human immunodeficiency virus type 1. F Clin Microbiol 1994;32: 2641-5.

8 Cassol S, Salas T, Gill MJ, et al. Stability of dried blood spot specimens for detection of human immunodeficiency virus 30:3039-42.

9 World Health Organisation. Acute respiratory infections in children: case management in small hospitals in developing countries. Geneva: WHO, 1990. (WHO/ARI/90.5)

10 Kanyama I, Kaona F, Siziya S, Mmiro F, Mirembe F, Bagenda D. Final report of the risk of occupational exposure to HIV infection among midwives and traditional birth attendants. Ndola: Tropical Diseases Research Centre, 1994.

11 Hira SK, Kamanga J, Brat GJ, et al. Perinatal transmission of HIV1 in Zambia. BMF 1989;299:1250-2. 12National AIDS prevention and control programme. Work plan and budget for 1993. Lusaka: Ministry of Health, 1992.

13 Lambert HL, Friesen H. Clinical features of paediatric AIDS in Uganda. Ann Trop Paediatr 1989;9:1-5. 